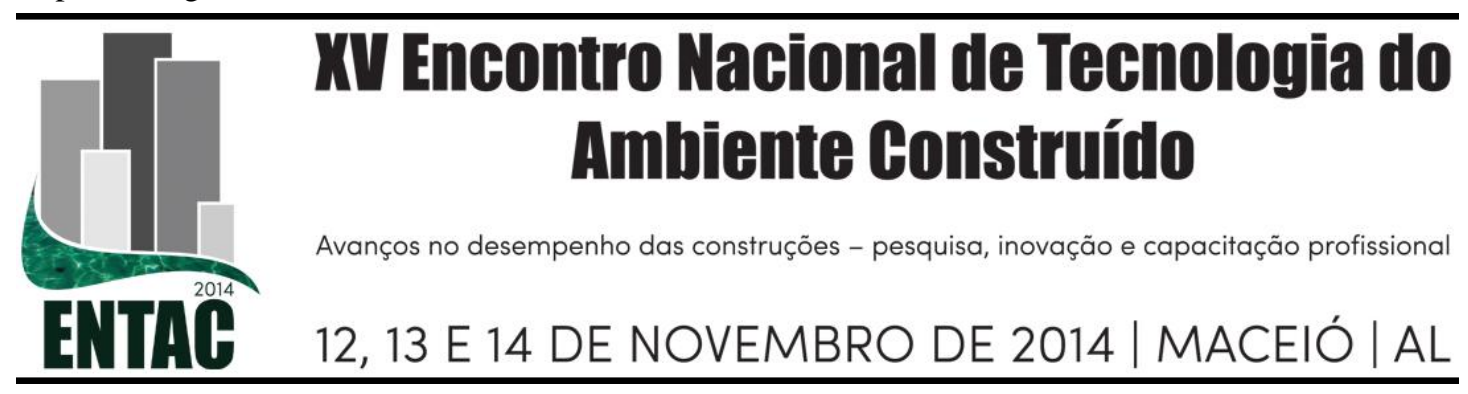

\title{
PANORAMA DO ZONEAMENTO BIOCLIMATICO NAS AMÉRICAS
}

\author{
WALSH, Angélica (1); LABAKI, Lucila (2); CÓSTOLA, Daniel (3) \\ (1)Fec UNICAMP, e-mail: angewalsh7@ hotmail.com (2)Fec UNICAMP, e-mail: lucila@ fec.unicamp.br, \\ (3)Fec UNICAMP, e-mail: daniel.costola@gmail.com
}

\begin{abstract}
RESUMO
O zoneamento bioclimático é o resultado da comparação entre as condições climáticas locais e as condições desejáveis de conforto, como base para a criação de recomendações de desenho arquitetônico. Trata-se de um trabalho complexo, no entanto, imprescindível para promover uma arquitetura adequada a seu entorno. As metodologias usadas para este fim são diversas e dependem de vários fatores, tais como as características geográficas preponderantes de um lugar, a disponibilidade e qualidade de informação climática, bem como de ferramentas de simulação computacionais acessíveis. $\mathrm{O}$ presente trabalho pretende realizar uma análise comparativa das propostas de zoneamento bioclimático desenvolvidas no continente americano com a finalidade de identificar as metodologias usadas, e sua aplicabilidade para a região centro-americana. Para esta identificação foram realizadas entrevistas com pesquisadores nacionais e internacionais, levantamento bibliográfico de normativas construtivas de cada país e selecionadas aquelas que apresentam uma proposta de zoneamento. Como resultado foi possível identificar dezesseis propostas de zoneamento em doze países, cinco das quais são de caráter normativo. Das dezesseis propostas estudadas, foram identificadas e analisadas três metodologias predominantes, a carta bioclimática de Givoni, o método de cálculo de graus-dia e a correlação entre a temperatura efetiva corrigida e outros índices de conforto. Também foram identificados outros critérios que eventualmente complementaram o zoneamento bioclimático. O método de cálculo de graus-dia de conforto foi o mais utilizado, porém, sua aplicabilidade demonstra limitações no momento em que outros critérios não são levados em conta. Por essa razão, foi complementado ocasionalmente com outras variáveis como a umidade e radiação solar, dando um resultado mais adequado. Para concluir, considera-se que o presente trabalho oferece uma visão abrangente do cenário atual no território americano no que diz respeito às metodologias de classificação de zoneamento bioclimático e seus desafios.
\end{abstract}

Palavras-chave: Zoneamento bioclimático, conforto térmico, eficiência energética.

\begin{abstract}
Climatic zoning occurs because of climatic conditions and the comfort desired requirements, as the base to establish energy efficiency design guidelines. The process of climatic zoning is a complex subject; however, it is essential in order to promote a bioclimatic architecture framework. The methods used for this purpose are diverse, and depend on several factors, such as the main geographic characteristics of a place, climate data easier to use and the quality, as well as simulation tool availability. This work tends to evolve a comparative analysis of climatic zoning propositions developed in America in order to identify the methods used as well as their applicability to the Central American region. For this identification, interviews were conducted, as well as literature review and the study of building energy regulations, codes of each country, and selected the ones that contain a climatic zoning. As a result, it was possible to identify sixteen climatic zoning proposals in twelve countries, five of which are normative. The main methodologies identified were: Givoni bioclimatic chart, degree-days method, and corrected effective temperature correlated with others thermal indices. Others criterias that eventually complemented the climatic zoning process, were also identified. Degree days method has been the most frequently applied, even though, its applicability shows some limitations due to absence of other elemental variables that
\end{abstract}


influence thermal performance of buildings. For this reason, it was complemented in some cases with parameters like solar radiation and humidity in order to provide a better result. To conclude, this work it's considered to provide a wide perspective of the current American scenario related to climatic zoning methods and its challenges.

Keywords: Climatic zoning, thermal comfort, energy efficiency.

\section{INTRODUÇÃO}

O zoneamento bioclimático é determinante na implementação de medidas de eficiência energética de edificações, já que contribui para identificar as características climáticas que influenciam mais fortemente o desempenho térmico e as necessidades de conforto na arquitetura.

De acordo com dados da International Energy Agency (IEA, 2011b) a energia consumida para o condicionamento ambiental e o aquecimento da água é responsável aproximadamente pela metade do consumo energético total dos edifícios. Esses dados variam entre países e entre tipologias arquitetônicas devido a diversos padrões de consumo e condições climáticas.

Para garantir o estabelecimento de bons parâmetros que permitam minimizar a dependência energética para o condicionamento ambiental, é fundamental aplicar um método de zoneamento bioclimático capaz de correlacionar a complexidade do clima em meso escala com as condições desejáveis de conforto dos usuários. No entanto, na prática é uma tarefa difícil, devido a muitos fatores, tais como a natureza dinâmica e multidimensional do clima.

Por estes motivos, muitos países têm investido grandes esforços em estudar e caracterizar o clima como base para o estabelecimento de normas de desempenho térmico. As abordagens adotadas por cada país são definidas de acordo com o contexto socioeconômico e podem ser focadas em soluções de baixo custo, principalmente estratégias passivas, ou as de condicionamento artificial, estratégias ativas.

No continente Americano, as habitações de Interesse social foram o principal foco nos primeiros trabalhos de zoneamento realizados na Argentina (1981), no Chile (1982) e no Brasil (2005). Estes se centralizaram na procura de soluções passivas e tornaram normas de desempenho térmico. Hoje, existem zoneamentos bioclimáticos utilizados como base de códigos de eficiência energética aplicados a outras tipologias tanto comerciais como residenciais em países da América do Norte e em Porto Rico, onde a abordagem de eficiência se baseia em estratégias tanto passivas quanto ativas.

Recentemente, o Standard 169, Climatic Data for Building Design Standards da ASRHAE 2013, foi atualizado sob o projeto 'Updating the ASHRAE Climatic Data for Design and Standards'. Esta revisão inclui informação climática de 5.564 lugares no mundo, as quais são classificadas em 9 grandes grupos e 27 subgrupos. Uma categoria climática para climas extremamente quentes foi adicionada nesta nova versão. Como resultado desse trabalho, foi gerado um mapa de zoneamento bioclimático de abrangência mundial, o qual é utilizado em Porto Rico, México e Canadá. (ASHRAE, 2013)

Estas propostas de Zoneamento bioclimático têm sido desenvolvidas com o uso de metodologias muito diversas, como as cartas psicrométricas de Givoni (1992), o cálculo de graus-dias (CIBSE, 2006), o cruzamento de variáveis climáticas, faixas de temperaturas, a aplicação de indicadores de conforto como a temperatura efetiva corrigida (TEC), o Índice de Belding Hatch (IBH), entre outros (IRAM 11603, 2011). 
Estes métodos, muitas vezes são insuficientes, e têm sido complementados com outras variáveis climáticas, como a amplitude térmica, altitude, umidade e radiação solar.

É notável, pela quantidade de variações nos métodos identificados, que hoje não existe consenso estabelecido sobre qual é o mais efetivo, indicando a necessidade de desenvolver mais pesquisas sobre o tema e criar assim ferramentas que permitam implementar políticas de eficiência energética para o setor de edificações.

Estudos prévios sobre este tema foram realizados e publicados em 2003 pelo Pacific Northwest National Laboratory (PNNL), onde se explica detalhadamente como foi gerado o zoneamento bioclimático dos Estados Unidos, o qual logo foi adotado pela ASHRAE e o International Energy Conservation Code em 2004. Uma comparação no contexto norte-americano é realizada entre diversas versões de zoneamento aplicadas à códigos de eficiência energética prévios. Depois de serem aplicadas diversas metodologias, como a análise hierárquica de Clusters, chegou-se a conclusão de que os critérios deviam ser mais subjetivos e simplificados para atingir os objetivos preestabelecidos no estudo (BRIGGS; TAYLOR; LUCAS, 2003). A metodologia resultante é a base do Standard 169-2013 anteriormente mencionado.

Publicações realizadas por Evans (2004) apresentam uma visão global do zoneamento bioclimático na América Latina, indicando os métodos e critérios predominantes para seis países da região. Ressalta, ao mesmo tempo, os aspectos mais relevantes no processo de zoneamento e descreve as características geográficas preponderantes de países situados na América central e no Caribe frente à edificação. Evans (2004) define o zoneamento bioclimático como a distribuição geográfica de fatores climáticos que afetam o desenho arquitetônico, e permite o estabelecimento de diretrizes de desenho bioclimáticas relevantes.

Por outro lado, se conhecem novas iniciativas de revisão dos zoneamentos no Chile (BUSTAMANTE, 2014) e no Brasil (RORIZ, 2012). Esta necessidade se fundamenta, em grande parte, na maior disponibilidade de dados climáticos, na experiência resultante de nove a trinta anos de implementação das normas anteriores e na rápida evolução das ferramentas de simulação térmica, o que não apenas permite, mas também exige hoje uma abordagem diferente para este trabalho.

Para o conhecimento dos autores, não existe um trabalho que proporcione uma visão abrangente das questões vinculadas a metodologias para zoneamento bioclimático para finalidades de edificações que levem em conta esses fatores.

Diante do exposto, o presente trabalho pretende analisar as propostas de zoneamento bioclimático desenvolvidas no continente americano, com a finalidade de identificar as metodologias usadas e sua aplicabilidade na região centro-americana.

Para atingir este objetivo, foram levantados artigos científicos e normas construtivas relacionadas ao desempenho energético de edificações e foram selecionadas aquelas que apresentam uma proposta de zoneamento bioclimático. Este trabalho foi complementado com entrevistas realizadas com arquitetos pesquisadores do Brasil, Colômbia, Venezuela e Cuba.

A seguir é exposta a metodologia implementada. Logo após, estão apresentados os resultados divididos em duas partes; a primeira, que aborda uma descrição dos países cujas metodologias de zoneamento foram identificadas e posteriormente os métodos de zoneamento mais usados atualmente e suas vantagens e desvantagens. Em seguida, outras variáveis climáticas identificadas e posteriormente estão descritas as conclusões. 


\section{MÉTODOLOGIA}

Este estudo se vale de uma pesquisa documental, complementada com entrevistas com quatro arquitetos pesquisadores na área da Arquitetura bioclimática do Brasil, Venezuela, Colômbia e Cuba.

A área de estudo compreendida neste trabalho é o continente americano, responsável por $41 \%$ da energia elétrica consumida no mundo (IEA, 2011a).

Foram estudadas as normas de eficiência energética para o setor de edificações disponíveis internacionalmente, e foram selecionados aqueles países onde existe um zoneamento bioclimático. Estas foram classificadas segundo seu status legal, com base no trabalho de Janda (2009) como obrigatórias, voluntárias, propostas e mistas.

Uma ênfase especial foi dada aos países cujas metodologias foram publicadas em artigos internacionais ou cujas documentações foram publicadas oficialmente como base de normas de desempenho. $\mathrm{O}$ estudo foi focado nos métodos para gerar as zonas climáticas e não para gerar as estratégias bioclimáticas resultantes, no entanto, em alguns casos, são utilizados para ilustrar as comparações entre metodologias.

\section{RESULTADOS}

\subsection{Países da América com zoneamento bioclimático}

Como resultado deste estudo, foi possível identificar dezesseis propostas de zoneamento bioclimático em doze países da América, como indicado no Quadro 1. Uma breve descrição do zoneamento de cada país é apresentada abaixo.

Quadro1 Países com zoneamento bioclimático para fins de edificações

\begin{tabular}{|c|c|c|c|c|}
\hline País & Ano & Tipo & Referência & Núm. de zonas \\
\hline \multirow[t]{2}{*}{ Chile } & 1977 & \multirow[t]{2}{*}{ Obrigatória } & NCh1079/2008 (MINVU, 2008) & 9 \\
\hline & 2000 & & $\begin{array}{c}\text { Artículo } 4.1 .10 \text { de la O.G.U } \\
\text { (MINVU, 2006) }\end{array}$ & 7 \\
\hline Argentina & 1981 & Obrigatória & IRAM 11603 (IRAM, 2011) & $\begin{array}{c}6 \\
12 \text { subzonas }\end{array}$ \\
\hline Uruguai & 1999 & Obrigatória & UNIT 1026:1999 (UNIT, 1999) & 3 \\
\hline EUA & 2004 & Misto & IECC, $2012($ ICC, 2011) & $\begin{array}{c}8 \\
24 \text { subzonas }\end{array}$ \\
\hline \multirow[t]{2}{*}{ Canadá } & 2011 & Obrigatória & NECB 2011 (CHBA,2011) & 6 \\
\hline & 2010 & Voluntário & IECC 2012 (ICC, 2011) & 6 \\
\hline \multirow[t]{2}{*}{ México } & 2009 & Voluntário & $\begin{array}{l}\text { NMX-C460-ONNCCE-2009 } \\
\text { (ONNCCE, 2008) }\end{array}$ & 8 \\
\hline & 2004 & Proposto & $\begin{array}{l}\text { (MORILLON; SALDANA; } \\
\text { TEJEDA, 2004) }\end{array}$ & 3 \\
\hline \multirow{2}{*}{ Brasil } & 2005 & Obrigatória & (ABNT NBR 15220, 2005.) & 8 \\
\hline & 2012 & Proposto & (RORIZ, 2012,2014) & 8,10 e 12 \\
\hline Peru & 2005 & Proposto & (RAYTER, 2008) & 9 \\
\hline Porto Rico & 2007 & Misto & IECC, 2012, (ICC, 2011) & $\begin{array}{c}2 \\
3 \text { subzonas }\end{array}$ \\
\hline Bolívia & 2007 & Trab. Academ. & (NAVARRO, 2007) & 8 \\
\hline Equador & 2011 & Voluntário & NEC-11, (MIDUVI,2011) & 6 \\
\hline Colômbia & 2012 & Proposta & (MADS, 2012) & 4 \\
\hline
\end{tabular}

Fonte: Autoria própria. 


\section{Argentina}

Os primeiros trabalhos de zoneamento bioclimático na América Latina foram desenvolvidos na América do Sul. Segundo Evans (2004), a Argentina foi o primeiro país da América Latina que desenvolveu um zoneamento bioclimático, o qual entrou em vigência em 1981 sob a denominação de "Clasificación ambiental de la República Argentina IRAM 11.603". Esta foi ajustada, posteriormente, nos anos de 1996 e 2001 sem mudanças essenciais no conteúdo.

O critério usado para determinar as zonas bioclimáticas se baseia em três indicadores: 1)Graus-dia: indicador de demanda de calefação no inverno; 2)Temperatura efetiva corrigida (TEC), correlacionada com os índices Belding e Hatch (IBH,1955) e o Voto Médio Estimado (VMP): indicadores de conforto no verão; 3)Amplitude térmica, como indicador de necessidade de incorporar inercia térmica ao edifício.

Segundo Evans (2004), o cálculo dos graus-dia no inverno é o indicador chave para a duração e severidade do período de aquecimento o qual aumenta de norte a sul. Assim como nas zonas de maior altitude. Como indicador de verão, optou-se pela TEC correlacionada com o IBH e VMP para um dia típico, a qual aumenta de sul a norte e na direção do litoral. Foram assim criadas seis zonas climáticas, as quais foram divididas em catorze subzonas a partir do indicador de amplitude térmica e a latitude para os climas mais frios.

\section{Chile}

Chile é considerado hoje como um dos países pioneiros em eficiência energética da América Latina. Este tem duas classificações climáticas para fins de edificações. $\mathrm{O}$ primeiro, conhecido como zoneamento climático habitacional do Chile, foi aprovado e declarado uma norma em outubro de 1982, sob o titulo de "Arquitectura y Construcción -Zonificación Climática Habitacional para Chile y recomendaciones para el diseño arquitectónico". Consta de nove zonas climáticas e foi criado a partir dos seguintes critérios: temperaturas, amplitude térmica, radiação solar, vento, precipitação, umidade e vegetação.

A segunda proposta, baseada na norma Térmica Chilena, entrou em vigência a partir do ano 2000, a cargo da "Ordenanza General de Urbanismo y Construcciones (OGUC)". Esta proposta, não substituiu a anterior, e baseia-se no cálculo de graus-dia de aquecimento, dando como resultado sete zonas climáticas.

Hoje existe um anteprojeto de norma térmica NTM 011-1 2014, o qual foi submetido a consulta pública em maio do presente ano. Esta nova proposta, tenta unificar os mapas de zoneamento anteriores, para evitar ambiguidades nas exigências (BUSTAMANTE, 2014).

\section{Brasil}

A partir do "I Encontro Nacional sobre Normalização em Uso Racional de Energia e Conforto Ambiental em Edificações" realizado em Florianópolis em 1991, foi apresentada uma primeira versão do zoneamento bioclimático brasileiro. Foi a partir de 2005 que este zoneamento virou norma Nacional (ABNT NBR 15220, 2005). Seu objetivo era constituir um conjunto de recomendações e estratégias construtivas para a adequação climática de habitações de interesse social. O país foi dividido em oito zonas climáticas definidas com o uso da carta psicométrica adaptada a partir da carta de Givoni (1992). As tabelas de Mahoney foram aplicadas para gerar as recomendações bioclimáticas de desenho (RORIZ; GUISI; LAMBERTS, 2001). 
A primeira proposta do zoneamento brasileiro tem sido objeto de estudo de muitos autores, gerando críticas construtivas e em conjunto com os avanços tecnológicos e o aumento de disponibilidade de dados climáticos no território brasileiro, dão lugar ao desenvolvimento de uma segunda proposta, a qual está sendo desenvolvida pelo arquiteto Mauricio Roriz. A metodologia proposta para este estudo consiste em caracterizar o clima em função da temperatura do ar, e aplicar simulações energéticas para estabelecer as estratégias bioclimáticas (RORIZ, 2012).

\section{Estados Unidos da América (EUA)}

Nos Estados Unidos da América as primeiras classificações climáticas com finalidade de edificações foram desenvolvidas a partir de 1989, eram apresentadas em forma de tabelas e abrangiam um número reduzido de localidades (240 cidades/ASRHAE 90.11989). Ao mesmo tempo não existia consenso entre as diversas versões que existiam porque eram o resultado de quatro metodologias diferentes.

No ano 2003, foi publicada a proposta utilizada na atualidade. Esta tinha a finalidade de fornecer uma abordagem simplificada e consistente, necessária para a implementação de códigos energéticos. Foi baseada na análise de 4.775 estações climáticas dos Estados Unidos e estendida na sua última versão (Standard 690 2013) a mais de 5.564 lugares no mundo. Esta foi o trabalho de pesquisadores do Departamento de Energia dos Estados Unidos da América do Pacific Northwest National Laboratory (PNNL).

Os mapas resultantes foram adotados e incluídos pela primeira vez no suplemento do IECC em 2004. Ao mesmo tempo, foram incorporados pela ASHRAE $90.1 \mathrm{em}$ sua primeira edição em 2004.

O território dos EUA foi dividido em oito zonas climáticas as quais são subdivididas em três grupos A, B e C (a partir de critérios de umidade) gerando um total de vinte e quatro subzonas. Os principais critérios adotados são o cálculo de graus-dia e o nível de umidade a partir da classificação de Köppen. Outras variáveis como a radiação solar foram adotadas, porém não refletidas no mapa resultante.

\section{Canadá}

Canadá conta com duas classificações climáticas: uma incluída no IECC 2012 e a outra parte do código energético Nacional (NB2011). Esta última, divide o país em seis zonas climáticas com base no cálculo de graus-dia de esfriamento (CHBA, 2011).

\section{México}

No caso do México, foram identificadas mais de três propostas de zoneamento bioclimático realizadas a partir de metodologias diferentes. Neste trabalho, são apresentadas duas.

A primeira foi realizada pelo arquiteto Morillón, publicada em 2004. Sua metodologia foi baseada nas cartas psicométricas de Givoni (1976) e Olgyay(1963). O resultado foi à criação de três zonas climáticas, as quais são apresentadas para cada mês do ano. A partir desta classificação, foram geradas recomendações de desenho que abrangem questões urbanas e arquitetônicas (MORILLON; SALDANA; TEJEDA, 2004).

Existe outra proposta de classificação ambiental oficial, que serve de base para as normas de isolamento térmico no México (NMX-C-460); estas zonas foram criadas a partir da metodologia adotada pela ASRHAE 2004 (ONNCCE, 2008). 


\section{Peru}

Peru, conta com uma categorização climática adaptada a partir da classificação climática de Köppen (RAYTER, 2008). Esta foi complementada com dados de altitude, radiação solar, inversão térmica e arquitetura vernácula. O território peruano é dividido em nove zonas climáticas. A metodologia aplicada não se conhece detalhadamente.

\section{Equador e Colômbia}

Mais recentemente, foram conhecidos trabalhos publicados no Equador (2011), onde a norma para a construção NEC-11(2011), contém um capítulo destinado à eficiência energética no setor da construção. Neste documento, são estabelecidas recomendações arquitetônicas para cada zona identificada com base no mapa de temperatura média anual (1965-1999) (MIDUVI, 2011).

Na Colômbia optou-se pelo cruzamento de dados climáticos a partir de três variáveis: temperatura do ar, umidade relativa e altitude. Outras variáveis climáticas são levadas em consideração para estabelecer as recomendações de desenho tais como a radiação solar e os ventos, porém não são refletidas no mapa proposto. Como resultado da classificação, o território foi dividido em cinco zonas climáticas (MADS, 2012).

\subsection{MÉTODOS IDENTIFICADOS}

Das dezesseis propostas de zoneamento assinaladas no Quadro 2, foi possível identificar três métodos predominantes e dez indicadores ou variáveis utilizadas de diversas formas para complementar eventualmente o processo de zoneamento.

\section{Quadro 2 Metodologias de zoneamento aplicadas por país}

\begin{tabular}{|c|c|c|c|c|c|c|c|c|c|c|c|c|c|c|}
\hline \multicolumn{2}{|c|}{$\begin{array}{l}\text { País/ No. de } \\
\text { zoneamentos }\end{array}$} & \multicolumn{3}{|c|}{$\begin{array}{l}\text { Metodologias para diagnóstico climático } \\
\text { para fins de Eficiência energética e } \\
\text { Conforto térmico no edifício }\end{array}$} & \multicolumn{7}{|c|}{$\begin{array}{c}\text { Variáveis climáticas/ } \\
\text { adicionais }\end{array}$} & \multicolumn{3}{|c|}{$\begin{array}{c}\text { Outras } \\
\text { classificações }\end{array}$} \\
\hline & & 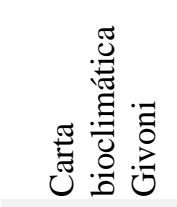 & 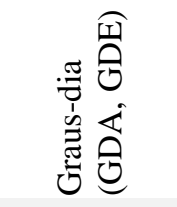 & 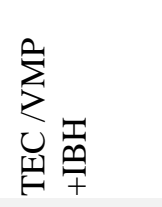 & $\stackrel{\stackrel{e}{E}}{\stackrel{D}{E}}$ & 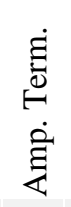 & 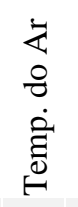 & 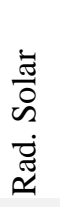 & $\frac{8}{\stackrel{0}{0}}$ & 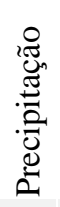 & 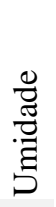 & 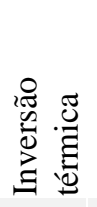 & 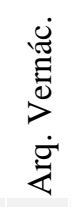 & 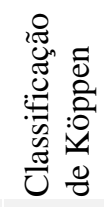 \\
\hline Chile & $\begin{array}{l}1 \\
2\end{array}$ & & $15^{\circ} \mathrm{C}$ & & & $\mathbf{x}$ & & $\mathbf{x}$ & $\mathbf{x}$ & $\mathbf{x}$ & & & & \\
\hline Argentina & 3 & & $15^{\circ} \mathrm{C}$ & $\mathbf{x}$ & & $\mathbf{x}$ & & & & & & & & \\
\hline Uruguai & 4 & & & $\mathbf{x}$ & & $\mathbf{x}$ & & & & & & & & \\
\hline EUA & 5 & & $15^{\circ} \mathrm{C} / 10^{\circ} \mathrm{C}$ & & & & $\mathbf{x}$ & $\mathbf{x}$ & & $\mathbf{x}$ & $\mathbf{x}$ & & & $\mathbf{x}$ \\
\hline Canadá & $\begin{array}{l}6 \\
7\end{array}$ & & $\begin{array}{c}15^{\circ} \mathrm{C} / 10^{\circ} \mathrm{C} \\
15^{\circ} \mathrm{C}\end{array}$ & & & & $\mathbf{x}$ & $\mathbf{x}$ & & $\mathbf{x}$ & $\mathbf{x}$ & & & $\mathbf{x}$ \\
\hline México & $\begin{array}{l}8 \\
9\end{array}$ & $\mathbf{x}$ & $15^{\circ} \mathrm{C} / 10^{\circ} \mathrm{C}$ & & & & $\mathbf{x}$ & $\mathbf{x}$ & & $\mathbf{x}$ & $\mathbf{x}$ & & & $\mathbf{x}$ \\
\hline Brasil & $\begin{array}{l}10 \\
11\end{array}$ & $\mathbf{x}$ & $18^{\circ} \mathrm{C} / 28^{\circ} \mathrm{C}$ & & & & & & & & & & & \\
\hline Peru & 12 & & & & $\mathbf{x}$ & & & $\mathbf{x}$ & & & & $\mathbf{x}$ & $\mathbf{x}$ & $\mathbf{x}$ \\
\hline Porto Rico & 13 & & $15^{\circ} \mathrm{C} / 10^{\circ} \mathrm{C}$ & & $\mathbf{x}$ & & $\mathbf{x}$ & $\mathbf{x}$ & & $\mathbf{x}$ & $\mathbf{x}$ & & & \\
\hline Bolívia & 14 & $\mathbf{x}$ & & & & & & & & & & & $\mathbf{x}$ & \\
\hline Equador & 15 & & & & & & $\mathbf{x}$ & & & & & & & \\
\hline Colômbia & 16 & & & & $\mathbf{x}$ & & $\mathbf{x}$ & & & & $\mathbf{x}$ & & & \\
\hline
\end{tabular}

Fonte: Autoria própria. 
O método identificado com maior frequência foi o cálculo de graus-dia. Este foi aplicado em oito casos, principalmente em climas predominantemente frios, onde as necessidades de aquecimento prevalecem.

\subsubsection{GRAUS-DIAS DE AQUECIMENTO OU ESFRIAMENTO}

O cálculo de graus-dia parte da hipótese que as necessidades de esfriamento e aquecimento variam linearmente com as diferenças de temperatura de base $\mathrm{e}$ temperaturas externas. Sua aplicação é considerada simples, devido a necessidade de se ter acesso apenas aos dados de temperaturas do ar, o que, ao mesmo tempo, reduz a probabilidade de erro.

A sua desvantagem consiste em que este método não leva em consideração os efeitos da umidade e radiação solar no consumo energético dos edifícios. Em casos como a Argentina, com mais de 15 anos de vigência da norma IRAM 11603, existem evidências que os graus-dias de aquecimento não representam por si só a severidade climática relativa de certas localidades do país (CZAJKOWSKI, 2007).

\subsubsection{CARTA PSICOMÉTRICA DE GIVONI}

A carta Psicométrica de Givoni (1992), baseada em trabalhos de Olgyay (1968) é uma ferramenta que correlaciona uma zona de conforto com parâmetros de umidade e temperatura do ar, gerando estratégias bioclimáticas para cada situação fora dessa zona. Esta tem sofrido algumas adaptações em países como o Brasil (RORIZ, 2002) para ser mais adequada às condições locais. A sua principal vantagem é a facilidade de aplicação e a quantidade reduzida de input necessária, porém desconsidera algumas variáveis climáticas importantes para o balanço térmico da edificação, como a radiação solar, a influência do vento.

A sua utilidade é indiscutível, no entanto tem algumas características que devem ser claramente definidas para evitar a geração de resultados inapropriados. A primeira questão é sobre a determinação adequada da zona de conforto. Esta depende de muitos fatores e varia segundo as localidades, pois o modelo de conforto mais recente e aceitado internacionalmente é o modelo adaptativo o qual não é considerado nesta ferramenta. A segunda questão é sobre a qualidade de dados climáticos utilizados, os quais deveriam ter horários ou pelo menos serem mais representativos a cada momento do dia.

\subsubsection{TEC+VMP +IBH}

A temperatura efetiva corrigida (BELFORD, 1940) é um índice de conforto resultado do efeito combinado da temperatura do bulbo seco, a temperatura de bulbo úmido, a temperatura radiante media e velocidade do ar. Este índice tem a vantagem de levar em conta uma serie de variáveis climáticas importantes para o desempenho térmico do edifício. A documentação consultada sobre a aplicação desta metodologia e sua correlação com os valores da IBH e VMP não apresenta uma explicação clara e detalhada, por este motivo a sua análise aprofundada não é apresentada neste artigo.

\subsubsection{VARIÁVEIS ADICIONAIS}

Outras variáveis climáticas como a amplitude térmica, radiação solar, vento, umidade, precipitação, altitude, e inversão térmica, são levadas em conta de diversas formas no processo de zoneamento. Uma síntese da influência das variáveis climáticas no desempenho térmico da edificação é apresentada por Evans (2004). 
Identificou-se que em alguns casos, estas complementam os métodos principais, como na Argentina e no Uruguai, onde a amplitude térmica é utilizada para subdividir as zonas devido a sua correlação com a necessidade de inércia térmica nos edifícios (IRAM, 2011).

\subsubsection{OUTROS FATORES}

Arquitetura vernácula é um tipo de arquitetura construtiva com técnicas regionais que guarda uma forte relação com o clima e a disponibilidade de materiais locais. Estas construções tornaram-se elementos caracterizadores dos lugares. (FERNANDES, 2012). Por este motivo, os trabalhos de zoneamento feitos no Peru e na Bolívia fundamentaram parte de seus métodos de classificação climática neste critério (NAVARRO, 2007).

A Classificação de Köppen Geiger é uma das classificações climáticas mais conhecidas internacionalmente. Embora este tipo de classificações não seja aplicável diretamente à arquitetura, tem uma relação indireta com a psicometria e consequentemente com o conforto térmico, devido principalmente à correlação que existe entre umidade, e pluviosidade e efetividade da precipitação. Esta relação é abordada por Briggs (2003) e apresentada como a justificação da utilização da classificação de Köppen Geiger para subdividir as zonas norte-americanas previamente agrupadas por faixas de graus-dia, em categorias A, B e C segundo o nível de umidade.

\section{CONCLUSÕES}

O estudo demonstrou que existe uma grande variedade de metodologias utilizadas na América para criar zonas bioclimáticas e que não existe um consenso na comunidade científica sobre qual é a mais adequada, indicando a necessidade de melhorar e atualizar os métodos atuais.

Identificou-se que as metodologias propostas em alguns casos, desconsideram aspectos climáticos relevantes para o desempenho bioclimático, o que foi justificado em muitas situações pela falta de dados climáticos suficientes e devido à complexidade que este trabalho representa.

O cálculo de graus-dia foi o método mais utilizado e aceitado internacionalmente para indicar o rigor climático, porém, sua aplicabilidade demostra limitações no momento em que outros critérios não são levados em conta.

Não é possível criar uma proposta de zoneamento para algo tão complexo e multidimensional como o clima aplicável a todas as situações e tipologias arquitetônicas. Razão pela qual é necessário delimitar claramente o propósito do trabalho.

\section{REFERÊNCIAS}

ASHRAE. AMERICAN SOCIETY OF HEATING, REFRIGERATING AND AIR CONDITIONING ENGINEERS, Standard 169-2013, Weather Data for Building Design Standards, Atlanta, 2013.

ABNT. ASSOCIAÇÃO BRASILEIRA DE NORMAS TÉCNICAS. NBR 15220-3 Informação e documentação - Referências - Elaboração. Rio de Janeiro, 2002.

BRIGGS R., TAYLOR Z.T., e LUCAS R. Climate Classification for Building Energy Codes and Standards. In: ASHRAE Winter Meeting, 2003, Chicago. Proceedings... Chicago, 2003.

BUSTAMANTE, W., Anteproyecto de norma NTM 011/2 2014. Requisitos y mecanismos de acreditación para acondicionamiento ambiental de las edificaciones, Santiago, 2014. 
CIBSE. CHARTERED INSTITUTION OF BUILDING SERVICES ENGINEERS. Degree-days: theory and application, CIBSE, Londres, 2006, 106p.

CZAJKOWSKI , J. Datos bioclimáticos para uso normativo en el diseño edilicio eficiente. Revisión de la normativa vigente y propuesta de mejoramiento y actualización, Avances en Energías Renovables y Medio Ambiente, Vol. 11, 2007.

EVANS, J. M. Zonificación bioambiental en Latinoamérica para una arquitectura sustentable, Avances en Energías Renovables y Medio Ambiente, Vol. 8, No 1, 2004.

FERNANDES, J. Princípios de sustentabilidade na arquitetura vernacular em Portugal. In: Congresso de construção 2012, 2012, Coimbra, Anais... Coimbra, 2012.

IRAM. INSTITUTO ARGENTINO DE NORMALIZACIÓN Y CERTIFICACIÓN. Norma IRAM 11603-2011 Acondicionamiento térmico de edificios Clasificación bioambiental de la República Argentina, Buenos Aires, 2011.

UNIT. INSTITUTO URUGUAYO DE NORMAS TÉCNICAS. UNIT 1026:1999: Aislamiento térmico en edificios. Zonificación climática, [s.n.], 1999.

ICC. INTERNATIONAL CODE COUNCIL. IECC 2012, Illinois, 2011.

IEA. INTERNATIONAL ENERGY AGENCY. Map energy Indicators, 2011a. Disponível em: http://www.iea.org/country/map_indicators/\# Acesso em: 16 maio 2014.

Technology Roadmap: Energy-efficient Buildings: Heating and Cooling Equipment. 2011b. Disponível em: http://www.iea.org/publications/ freepublications/publication/technology-roadmap-energy-efficient-buildings-heating-andcooling -equipment-insights.html, Acesso em: 16 maio 2014.

JANDA, K., Worldwide status of energy standards for buildings: a 2009 update. In: European council for an energy efficient economy Summer Study, 2008, Colle Sur Loop. Proceedings... ECEEE, Colle Sur Loop, 2009.

MADS. MINISTERIO DE AMBIENTE Y DESARROLLO SOSTENIBLE. Criterios ambientales para el diseño y construcción de vivienda urbana, Bogotá, 2012, 200p.

MIDUVI. MINISTERIO DE DESARROLLO URBANO Y VIVIENDA. NEC-11 Eficiencia Energética en la construcción en Ecuador, Quito, 2011.

MINVU. MINISTERIO DE LA VIVIENDA Y URBANISMO. Manual de Aplicación de la Reglamentación Térmica 2006, 1. ed. Santiago de Chile, 2006, 54p.

.NCh1079/2008, Norma chilena oficial de Zonificación Climático-Habitacional, Santiago de Chile, 2008.

RAYTER, D. Guía de aplicación de arquitectura bioclimática en locales educativos. ME, Lima, 2008, 114p.

MORILLON, D.; SALDANA, R.; TEJEDA, A., Human bioclimatic atlas for México. Solar Energy. Volume 76, n.6, Pages 781-792, 2004.

NAVARRO, F. Arquitetura e clima na Bolívia : Uma proposta de zoneamento, 2007. Tese (Mestrado)- Faculdade de Engenharia Civil, Arquitetura e Urbanismo, Universidade Estadual de Campinas, Campinas, 2007.

CHBA. CANADIAN HOME BUILDERS' ASSOCIATION. The National Energy Code for Buildings: Overview and Issues, 2011. Disponível em: http://www.chba.ca/uploads/trc /webinar/necb\%20overview\%20\%20issues.pdf Acesso em: 6 maio 2014.

ONNCCE. ORGANISMO NACIONAL DE NORMALIZACIÓN Y CERTIFICACIÓN DE LA CONSTRUCCIÓN Y EDIFICACIÓN. APROY-NMX-C-460-ONNCCE-2008, México DF., 2008.

RORIZ, M. Segunda proposta de revisão do Zoneamento bioclimático do Brasil. ANTAC Grupo de Trabalho sobre Conforto Ambiental e Eficiência Energética de Edificações. São Carlos, 2012.

RORIZ, M.; GUISI E.; LAMBERTS R. Um zoneamento bioclimático para a arquitetura no Brasil. In: Jornada sobre clima e aplicação na comunidade de países de língua oficial portuguesa, 2001, Maceió, Anais... Maceió: UFAL, 2001. 